\title{
Bispohosphonates in the Treatment of Breast Cancer
}

\author{
Chair: Tanja Fehm ${ }^{a}$ \\ Participants: Wolfgang Janni ${ }^{b}$ Eugen Ruckhäberle ${ }^{a} \quad$ Florian Schütz $^{c} \quad$ Erich-F. Solomayer $^{d}$ \\ ${ }^{a}$ Frauenklinik, Heinrich-Heine-Universität Düsseldorf, Germany; \\ ${ }^{b}$ Frauenklinik, Universitätsklinikum Ulm, Germany; \\ ${ }^{c}$ Universitätsfrauenklinik Heidelberg, Germany; \\ ${ }^{\mathrm{d}}$ Klinik für Frauenheilkunde, Geburtshilfe und Reproduktionsmedizin, Universitätsklinikum des Saarlandes, Homburg/Saar, Germany
}

\section{Question 1: In Which Cases Do You Offer Bisphosphonates in the Adjuvant Setting? How Long Do You Treat with Bisphophonates in the Adjuvant Setting?}

Janni: I disclose the current data to postmenopausal women, but I do not derive a recommendation from the data. Based on the controversial data, I leave the decision to the patients.

Ruckhäberle: We recommend bisphonates to many but not all postmenopausal patients in our tumor board. In low-risk cases and triplenegative postmenopausal patients a clear benefit has not been shown yet and is not recommended routinely. In premenopausal patients only selected cases receive a recommendation for adjuvant bisphonates (e.g. patients fullfilling criteria of ABCSG 12).

Schütz: I routinely discuss an adjuvant bisphosphonate treatment with my postmenopausal patients, especially if they are on therapy with an aromatase inhibitor. However, in premenopausal patients I use bisphosphonates only in patients with osteoporosis.

Solomayer: From an oncological point of view we discuss an adjuvant bisphosphonate therapy with our postmenopausal patients, especially if they are under treatment with aromatase inhibitors and/or suffer from osteopenia/osteoporosis. We advise an adjuvant bisphosphonate therapy to premenopausal patients only in selected cases.

\section{Question 2: What Is Your Explanation that Bisphosphonates Only Work in Postmenopausal Women?}

Janni: We have very limited research to speculate on a solid basis, we might never know. Based on the data on disseminated tumor cells in bone marrow, we can presume that the bone marrow is host to dormant tumor cells for a long time. Changes in the microenvironment by bisphosphosphonates, inhibiting the tumor cells' ability to proliferate, might play a stronger role in an estrogen deprivation status.

Ruckhäberle: In my interpretation of the data, bisphosphonates do not only work in postmenopausal patients, but so far we are unable to detect premenopausals who would benefit from bisphosphonate treatment. In my opinion changes in the micromilieu of the bone and bone marrow are responsible for the success. On the other hand I speculate that treatment of a beginning osteporosis could stabilize bone and thus the general condition of the patients and patient's immune system. The risk of an undiagnosed osteoprosis is much higher in postmenopausal patients, supporting the so called 'low estrogen milieu' hypotheis.

Schütz: My hypothesis is that bisphosphonates have a high impact on the bone marrow's microenvironment. Cytokines as well as chemokines seem to play a big role in hosting disseminated tumor cells. By using bisphosphonates we influence the cytokine balance and may initiate a tumor cell hostile environment that leads to a longer dormancy of tumor cells. Our long-time follow-up data have shown that the protective effect could be observed in the treatment time only. After more than 10 years we have seen identical recurrence rates in treated and untreated patients.

\section{KARGER \\ Fax +497614520714

\section{() 2015 S. Karger GmbH, Freiburg}

1661-3791/15/0103-0220\$39.50/0
Prof. Dr. Tanja Fehm

Department of Obstetrics and Gynecology

Heinrich-Heine-University Düsseldorf

Moorenstrasse 5, 40225 Düsseldorf, Germany

tanja.fehm@med.uni-duesseldorf.de 
Solomayer: Changes in the mircoenvironment of the bone marrow because of low estrogen levels and an inferior immune system - compared to premenopausal women - might be an explanation why bisphosphonates show a higher impact / positive influence on postmenopausal women.

\section{Question 3: Which Patients Do You Treat with Bisphosphonates in the Metastatic Setting Since Denosumab Has Been Established as an Alternative to Bisphosphonates?}

Janni: Bisphosphonates are, in my view, still a treatment option for patients preferring oral medication, with progressive disease on denosumab, or in settings with increased pressure on cost effectiveness.

Ruckhäberle: Patients who are stable on bisphosphonates for a long time and who do not suffer from side effects stay on bisphosphonates. All newly diagnosed patients with bone metastases receive denosumab. When patients deny intraveonous or subcutaneous treatment they receive commonly oral ibandronate. In the clinical setting bisphosphonates are preferred because of the costs.

Schütz: Bisphosphonates are very effective in treating metastatic breast cancer. However, denosumab has shown a little bit more effectiveness in some patients and a different toxicity profile. In my opinion, bisphosphonates should be used in those patients who prefer tablets instead of intravenous application or if hypocalcemia is a concern.

Solomayer: Patients who are already on treatment with bisphosphonates and do not suffer from severe side effects should stay on treatment with bisphosphonates. Otherwise denosumab should be preferred. Another reason to give bisphosphonates is the patient's wish for oral medication instead of intravenous or subcutaneous injections, or progressive disease on therapy with denosumab.

\section{Question 4: How Long Do You Treat in the Metastatic Setting? Do You Extend the drug Intervall to Reduce Side Effects?}

Janni: I basically treat livelong in patients with bone metastases. After a long period of stable disease, e.g. for more than 2 years, I start to extend drug intervals.

Ruckhäberle: Recommendations and trial data exist for 1-2 years of treatment. If patients do well under therapy, show no severe side effects I usually treat livelong. In stable disease situations after 2 years of treatment, therapy breaks or longer therapy intervalls (e.g. every 3 months) are feasible.

Schütz: Bone targeted treatment in the metastatic setting is a supportive treatment that should not be stopped. However, in pa- tients that are treated more than 1-2 years I think about longer intervals between the applications (e.g. prolonging from every 4 to every 12 weeks in intraveneous bisphosphonates). In patients on denosumab I do not prolong intervals, as the antibody is not stored in bone like bisphosphonates.

Solomayer: If they experience no severe side effects or progressive disease patients should be treated livelong. After a period of stable disease, e.g. $>2$ years, extended therapy intervals can be discussed with the patient.

\section{Question 5: Would You Change from Bisphosphonates to Denosumab and Vice Versa in Case of Progression?}

Janni: Yes, even though we have to admit that the evidence basis for this strategy is very limited.

Ruckhäberle: I do change from bisphosponate to denosumab in case of severe side effects or progression, keeping in mind that there is no clearly supporting data. In patients who started with denosumab I do switch to bisphosphonates in cases with progession or if patients prefer tablets.

Schütz: I change from bisphosphonates to denosumab in case of progression as denosumab may be more effective. However, I only do change from denosumab to bisphosphonates in patients with intolerable toxicity on the antibody.

Solomayer: We change from bisphoshonates to denosumab in case of progression. Vice versa only in cases of severe toxicity.

\section{Participants}

\author{
Univ.-Prof. Dr. W. Janni \\ Frauenklinik \\ Universitätsklinikum Ulm \\ Prittwitzstr. 43, 89075 Ulm, Germany \\ wolfgang.janni@uniklinik-ulm.de \\ Dr. Eugen Ruckhäberle \\ Department of Obstetrics and Gynecology \\ Heinrich-Heine-University Düsseldorf \\ Moorenstrasse 5, 40225 Düsseldorf, Germany \\ eugen.ruckhaeberle@med.uni-duesseldorf.de
}

Prof. Dr. med. Florian Schütz

Universitätsfrauenklinik Heidelberg

Im Neuenheimer Feld 440, 69120 Heidelberg, Germany

florian_schuetz@med.uni-heidelberg.de

Prof. Dr. Erich-F. Solomayer

Klinik für Frauenheilkunde, Geburtshilfe und Reproduktionsmedizin

Universitätsklinikum des Saarlandes

66421 Homburg/Saar, Germany

erich.solomayer@uks.eu 